\title{
THEORETICAL INVESTIGATIONS OF OSCILLATIONS OF ROOT CROP HEAD CLEANER HANGED ON INTEGRAL ROW-CROP TRACTOR
}

\author{
VolodymyrBulgakov $^{1}$, Stanislav Nikolaenko ${ }^{1}$, Ivan Holovach ${ }^{1}$, \\ Semjons Ivanovs ${ }^{2}$, Kaspars Vartukapteinis ${ }^{2}$ \\ ${ }^{1}$ NationalUniversity of Life and Environmental Sciences of Ukraine, Ukraine \\ ${ }^{2}$ Latvia University of Agriculture, Latvia \\ semjons@apollo.lv
}

\begin{abstract}
There is proposed a new design of a trailed sugar beet head cleaner from the remnants of tops without extraction of beets from the soil, with a vertical cleaning shaft. However, the quality of its operation depends to a large extent on the magnitude of the vertical oscillations of the cleaner frame, which take place at a high speed of its forward movement. According to the developed equivalent scheme and the initial equations in the form of Lagrange of the 2nd kind, a mathematical model has been constructed for the root crop head cleaner, hanged from behind to an integral tractor, consisting of two nonlinear differential equations describing the oscillations of its pneumatic tracer wheels and the cleaning tool when moving along the surface irregularities. Numerical simulation on the PC has made it possible to determine the optimal design and kinematic parameters of the cleaner, allowing to reduce the magnitude of oscillations of its tracer wheels, frame and cleaning tool, which, on the whole, significantly increases the efficiency and quality of the root crop cleaning from the tops without extracting the root crops from the soil.
\end{abstract}

Keywords: sugar beet, harvesting, cleaner, oscillations.

\section{Introduction}

Sugar beet is an important agricultural crop used for the production of sugar; and it also has great prospects for the production of bioenergy [1]. The leading producers of sugar beet in Europe are Ukraine, Germany, France and Poland. Contemporary technologies of harvesting sugar beet tops assume, at first, complete uncopied cutting of the main green mass with subsequent individual postcleaning of the heads of root crops from the remnants of the foliage [2]. The operation of additional cleaning of the root crop heads from the remnants of tops (leaves) without extracting the heads from the soil should meet high demands for the quality of removal of the green and dry residues from the surfaces of the heads, excluding damage caused by the additional cleaning tools. We have developed a new design of a trailed cleaner of sugar beet heads from the remnants of tops (leaves), with a vertical cleaning shaft. However, the quality of its operation depends to a large extent on the magnitude of the vertical oscillations of the cleaner frame, which take place at a high speed of its forwards movement. The use of pneumatic tracer wheels causes intensive oscillations of the root crop head cleaner in a vertical plane, which have the greatest effect on the quality of this technological process. In this connection, a need arises for theoretical investigation of the movement of a root crop head cleaner attached to the wheeled integral row-crop aggregating tractor and for determining the degree of influence of its structural and kinematic parameters on the vibrations in the vertical plane. The method of constructing computational mathematical models of agricultural machines is described in detail in [3]. A methodology of constructing computational mathematical models of agricultural machines is described in detail in [3], in which problems related to simulation of the plane-parallel movement of aggregates, their oscillations in the longitudinal-vertical plane, as well as the dynamics of drives of the machine units performing certain technological processes are considered. However, there is still a need to improve the methods of theoretical research, the development of appropriate numerical calculation programs and the possibilities of wide simulation on a PC, in order to find optimal design and kinematic parameters. The development of a contemporary beet harvesting technology is connected with the development and wide application of combined machines [4-8]. Thus, the use of integral row-crop tractors as energy resources makes it possible to widely use combined beet harvesting aggregates consisting of front-mounted top-harvesting machines that carry out a complete, uncopied cutting of the main mass of sugar beet tops and its collection for further use (for example, for biogas production), and rear-mounted root crop head cleaners from the remnants of the tops (leaves) [9]. Such an aggregate not only qualitatively performs the technological process of harvesting the tops, but is also considerably less energy-intensive (in contrast to the traditional harvesting methodologies of the tops). In the studies of oscillations of the front-mounted and rear-mounted combined machine aggregates moving along the soil surface irregularities the theory of oscillations of root crop head 
cleaners from the remnants of the tops without extracting the heads from the soil is not considered [10].

The purpose of this work is a theoretical study of the oscillations of the root crop head cleaner with the pneumatic tracer wheels moving along the soil surface irregularities and determination of the optimal design and kinematic parameters.

\section{Materials and methods}

During the research, methods for constructing computational mathematical models of agricultural machines were used, based on the compilation and solution of the systems of differential equations, as well as numerical simulation applying programs compiled on the PC.

Let us consider analytically the movement of the sugar beet head cleaner of the new design only in the longitudinally vertical plane, i.e. we will construct a mathematical model of oscillations of a cleaner, rear-mounted on the wheeled aggregating tractor, moving along the irregularities of the soil surface. The use of the rear-mounted cleaner leads in the course of work to its movements in space, which are determined by the forward speed of the tractor, the relief of the field surface in the inter-row aisle of the crops, the placement of the tracer wheels relative to the frame and the three-point linkage system (device for mounting machine for tractor), etc. (Figs 1, 2). At the same time, the wheels of the tractor, as well as the pneumatic tracer wheels of the cleaner, move along the inter-row aisles, between which the main mass of the leaves is cut off.

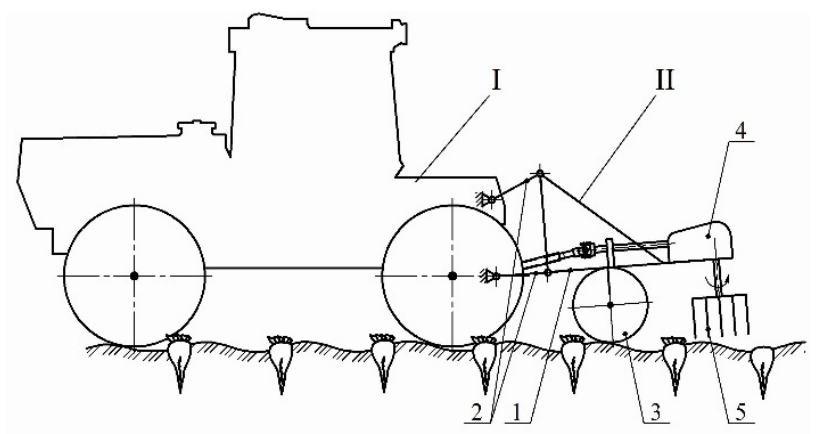

Fig. 1. Scheme of the aggregate: I - integral rowcrop tractor; II-rear-mounted cleaner of the root crop heads: 1 - frame; 2 - linkage system; 3 - tracer wheel; 4 - drive; 5 - cleaning tool

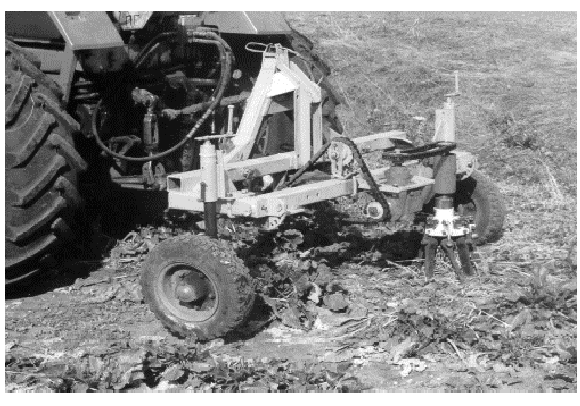
Fig. 2. Sugar Beet Head Cleaner with a
Vertical Cleaning Shaft

Let us compose an equivalent scheme of the root crop head cleaner, rear-mounted on the tractor, considering its movement in the longitudinal-vertical plane (Fig. 3). As it is evident from the diagramme, the frame of the root head cleaner from the top remnants is attached to the aggregating tractor by means of two lower links $B D$ and one upper link $L M$, which have pivots at points $B, D, L$ and $M$. In this case, vertical oscillations of the tractor during the operation of the aggregate are practically not transferred to the frame of the cleaner. While moving along the irregularities of the soil surface, the aggregating tractor acquires vertical oscillations. Therefore, the pivots at the points $D$ and $M$, as belonging to the tractor, also perform vertical oscillations. However, due to the presence of pivots at points $B$ and $L$ and, belonging to the frame of the cleaner, and a significant mass of the cleaner itself, these oscillations are practically not transferred to the cleaner frame.

The vertical oscillations of the tractor cause only angular vibrations of the links $B D$ and $L M$, connecting the frame of the cleaner to the tractor. At the same time, we can assume with a sufficient degree of accuracy that the links $B$ and $L$ do not perform vertical vibrations, but the links $B D$ and $L M$ only rotate around the points $B$ and $L$; and their other ends (pivots $D$ and $M$ ) oscillate together with the tractor. Thus, the vertical oscillations of the tractor are actually converted into angular oscillations of the links $B D$ and $L M$, not transmitted to the frame of the trailed cleaner. Therefore, we assume that the points of suspension of the cleaning frame (i.e. pivots $B$ and $L$ ) move rectilinearly and evenly. 


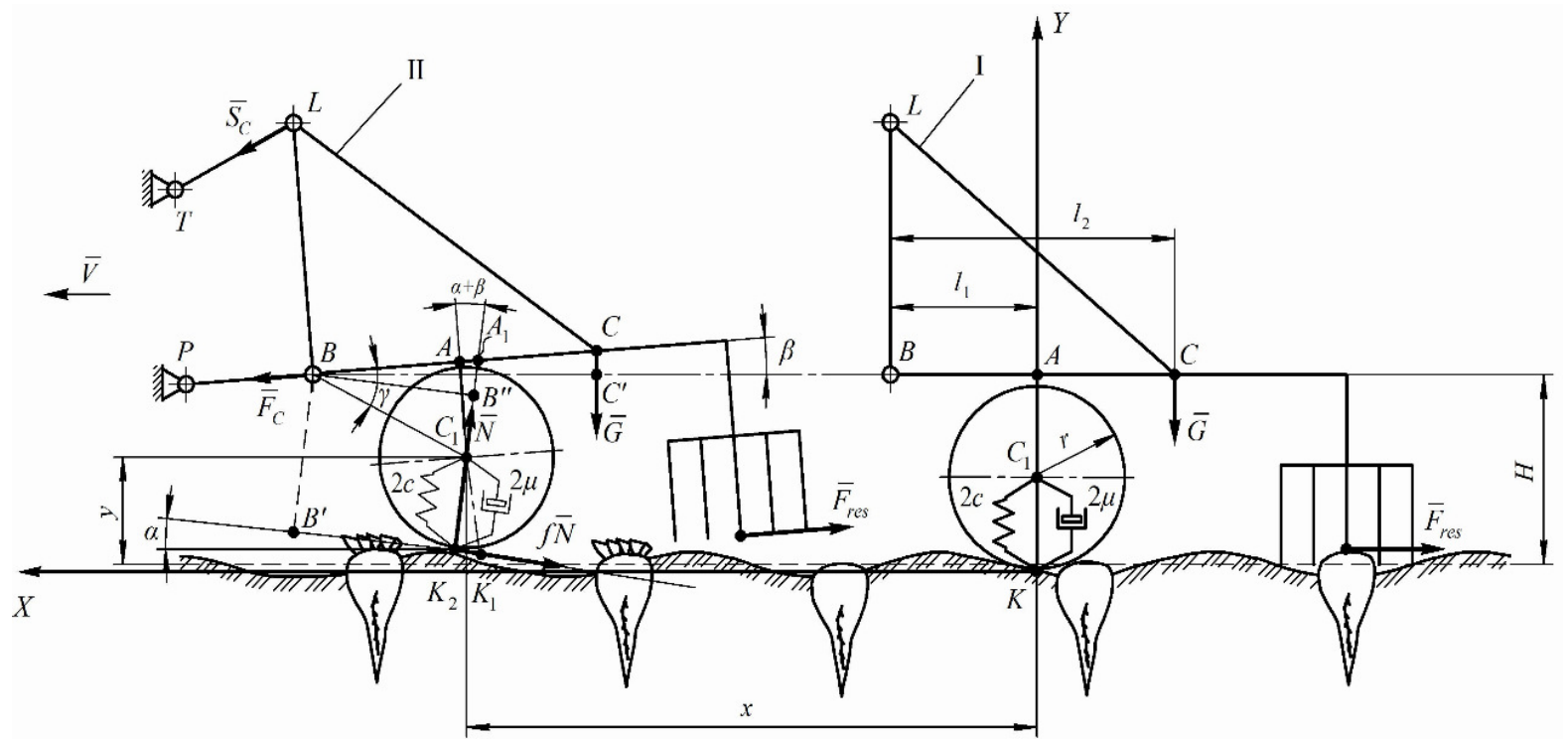

Fig. 3. Equivalent scheme of movement of the root crop head cleaner

Here we will consider the movement of only one of the tracer wheels of the cleaner with a radius $r$ (the second wheel is in the same state as the first one). Suppose that in the process of operation the tracer wheel, moving in the inter-crop aisles along the surface of the soil of a sinusoidal transverse profile [11], has passed from one position I to another position II. In the plane of rotation, the forces acting upon the tracer wheel are: the weight of the cleaner $\bar{G}$ and the tractive force $\bar{F}_{T}$, directed along the link $B D$ from the point $B$ to point $D$. Besides, along the link $L M$, there is an effort $\overline{S_{T}}$ directed from the point $L$ to the point $M$. From the side of the root crophead cleaner, a resistance force $\overline{F_{r e s}}$ acts at the point $E$, which is equal in magnitude and opposite in direction to the stripping force $\overline{Q_{b r u}}$ of the tops from the root crop heads, which will be defined below. Under the impact of these forces, a normal $\bar{N}$ and tangential $f \bar{N}$ reaction occurs at the point of contact $K_{1}$ between the tracer wheel and the irregularities of the soil surface (where $f$ is the rolling resistance coefficient). The direction of the action of the normal $\bar{N}$ and tangential $f \bar{N}$ reactions of the soil is determined by the angle $\alpha$. The angle between the traction force $\bar{F}_{T}$ and the direction of movement will be denoted by $\beta$, which determines the character of the vertical oscillations of the root crop head cleaner in the longitudinally vertical plane. Let us denote the height of the frame suspension by $H$. We will consider that the speed of the forward movement of this aggregate is constant and equal to $V_{M}$.

Let us regard the presented dynamical system with respect to the fixed Cartesian coordinates $K_{1} X Y$ [12], assuming that all its points are moving only in this plane. Let us locate the centre of the coordinate axes at the point $K_{1}$, i.e. at the point of contact of the tracer wheel with the soil. As can be seen from Fig. 3, the location of the centre of the tracer wheel is determined by the coordinates $x$ and $y$. The movement of the suspension points of the frame of the cleaner towards the aggregating tractor (points $B$ and $L$ ) is assumed to be rectilinear and even. In addition, the centre of masses of the root head cleaner (point $C$ ) is at a distance $l_{2}$ from the suspension point $B$. The distance between the axis of the cleaner (point $B$ ) and the axis of the installation of its tracer wheels (point $A$ ) will be denoted by $l_{1}$. We will denote the mass of the entire root head cleaner by $M$, the mass of the tracer wheels - by $m$ where $m=m_{1}+m_{2}$ ( $m_{1}$ is the mass of the first tracer wheel, $m_{2}-$ the mass of the second copying wheel). The force of the weight $\bar{G}$ of the examined root crop head cleaner will be considered as applied at its centre of masses (point $C$ ). The mass $m$ of the tracer wheels is concentrated in the point $C_{1}$.

Pneumatic tracer wheels are represented in the form of elastic damping models, which have a stiffness coefficient $c$ and a damping coefficient $\mu$. Since there are two tracer wheels, the indicated coefficients will be doubled. Let us assume that the tracer wheels are moving along the irregularities of the soil surface in the sinusoidal profile, which varies according to the following law [11]: 


$$
y=h\left[1+\sin \left(k x-\frac{\pi}{2}\right)\right]
$$

where $y$ - ordinate of the height of the soil surface irregularities, $\mathrm{m}$;

$h$ - half of the height of the soil surface irregularities, m;

$k$ - frequency of the soil surface irregularities, $\mathrm{m}^{-1}$;

$x=V_{M} \cdot t-$ value of the current coordinate, $\mathrm{m}$;

$V_{M}$ - speed of the movement of the cleaner, $\mathrm{m} \cdot \mathrm{s}^{-1}$;

$t$ - current time, s.

We consider that the tracer wheel is in contact with the soil surface irregularity at a point $K_{1}$ located on the sinusoid defined by the form (1). Since the centre of masses of the pneumatic tracer wheels, due to their damping properties, performs independent oscillatory movements (point $C_{1}$ ) and the ordinates of the heights $y$ of the soil surface irregularities is much smaller than the displacement value $x$ of the cleaner, we can assume that these oscillations will be determined by the independent coordinate $y$. The position of the centre of masses of the cleaner (point $C$ ), in the longitudinal-vertical plane, is completely determined by the independent coordinate $\beta$, i.e. the inclination angle of the cleaning frame to the horizon. Thus, the considered oscillatory system has two degrees of freedom, and its motion is entirely determined by two generalized coordinates:

$$
\left.\begin{array}{l}
q_{1}=\beta, \\
q_{2}=y .
\end{array}\right\}
$$

In this case, the speed $V$ of the centre of masses of the tracer wheels (point $C_{1}$ ) will be equal to:

$$
V=\sqrt{\dot{x}^{2}+\dot{y}^{2}} .
$$

Taking into account that $x=V_{M} \cdot t$, we obtain:

$$
V=\sqrt{V_{M}^{2}+\dot{y}^{2}} .
$$

The angular velocity of the tracer wheel, necessary to determine the kinetic energy of the given dynamical system, can be expressed in terms of the known parameters:

$$
\omega=\frac{d S}{d t} \cdot \frac{1}{r},
$$

where $\omega$-angular velocity of the tracer wheel, $\operatorname{rad} \cdot \mathrm{s}^{-1}$;

$S$ - value of the circular movement of the wheel along the surface profile of the soil, m; $r$ - radius of the tracer wheel, $\mathrm{m}$.

In this case, the differential of the movement of the tracer wheel $d S$ will be equal to:

$$
d S=\sqrt{d x^{2}+d y^{2}}=\sqrt{1+h^{2} k^{2} \cos ^{2}\left(k x-\frac{\pi}{2}\right)} d x,
$$

Or:

$$
d S=\sqrt{1+h^{2} k^{2} \cos ^{2}\left(k V_{M} \cdot t-\frac{\pi}{2}\right)} \cdot V_{M} d t .
$$

By substituting the value of (6) into (4), we obtain the value of the angular velocity:

$$
\omega=\frac{\sqrt{1+h^{2} k^{2} \cos ^{2}\left(k V_{M} \cdot t-\frac{\pi}{2}\right)} \cdot V_{M}}{r} \text {. }
$$


To compile differential equations of motion of the considered oscillatory system, we use the initial equations in the Lagrange form of the 2nd kind [3]:

$$
\frac{d}{d t}\left(\frac{\partial T}{\partial \dot{q}_{i}}\right)-\frac{\partial T}{\partial q_{i}}=Q_{i}-\frac{\partial P}{\partial q_{i}}-\frac{\partial R}{\partial \dot{q}_{i}},
$$

where $T$ - kinetic energy of the system under consideration;

$Q_{i}$ - generalised force;

$P$ - potential energy;

$R$ - dissipative function.

The kinetic energy $T$ of the considered dynamical system will be:

$$
T=\sum_{i=1}^{4} T_{i}=T_{1}+T_{2}+T_{3}+T_{4},
$$

where $T_{1}$ - kinetic energy of the forward movement of the cleaner (its centre of mass);

$T_{2}$ - kinetic energy of the oscillatory movement of the cleaner frame around the point $B$;

$T_{3}$ - kinetic energy of the rotational movement of the tracer wheels around their axis;

$T_{4}$ - kinetic energy of vertical oscillations of the pneumatic tracer wheels due to elasticviscous resistance of tires.

The components of the kinetic energy $T$, in turn, will be equal to:

$$
T_{1}=\frac{M V_{M}^{2}}{2},
$$

where $M-$ mass of the cleaner, $\mathrm{kg}$;

$V_{M}$ - speed of the forward movement of the centre of masses of the cleaner, $\mathrm{m} \cdot \mathrm{s}^{-1}$.

$$
T_{2}=\frac{I_{B} \dot{\beta}^{2}}{2},
$$

where $I_{B}$ - moment of inertia of the cleaner frame relative to the axis, which is perpendicular to the longitudinal-vertical plane and passes through the point $B, \mathrm{~kg} \cdot \mathrm{m}^{2}$;

$\dot{\beta}$ - angular velocity of rotation of the cleaner frame, $\mathrm{s}^{-1}$.

$$
T_{3}=\frac{I_{k} \omega^{2}}{2}=\frac{1}{2 r^{2}} I_{k} V_{M}^{2}\left[1+h^{2} k^{2} \sin ^{2}(k x)\right],
$$

where $I_{k}-$ moment of inertia of both tracer wheels relative to the axes of their rotation, $\mathrm{kg} \cdot \mathrm{m}^{2}$; $\omega$ - angular velocity of rotation of the tracer wheel, $\mathrm{s}^{-1}$.

$$
T_{4}=\frac{m \dot{y}^{2}}{2},
$$

where $m$ - mass of both tracer wheels, $\mathrm{kg}$;

$\dot{y}$ - speed of vertical oscillations of the tracer wheels, $\mathrm{m} \cdot \mathrm{s}^{-1}$.

Then, taking into account expressions (10) - (13), expression (9) takes the following form:

$$
T=\frac{M V_{M}^{2}}{2}+\frac{I_{B} \dot{\beta}^{2}}{2}+\frac{I_{k} \omega^{2}}{2}+\frac{m \dot{y}^{2}}{2},
$$

Or:

$$
T=\frac{M V_{M}^{2}}{2}+\frac{I_{B} \dot{\beta}^{2}}{2}+\frac{1}{2 r^{2}} I_{k} V_{M}^{2}\left[1+h^{2} k^{2} \sin ^{2}(k x)\right]+\frac{m \dot{y}^{2}}{2} .
$$

Let us define the necessary partial derivatives, which are provided by equation (8), for each of the generalised coordinates $\beta$ and $y$. According to the first generalized coordinate $\beta$, the partial derivative of the kinetic energy $T$ of the system will have the following appearance: 


$$
\frac{\partial T}{\partial \dot{\beta}}=I_{B} \dot{\beta}
$$

Then:

$$
\frac{d}{d t}\left(\frac{\partial T}{\partial \dot{\beta}}\right)=I_{B} \ddot{\beta}
$$

Considering that

$$
\frac{\partial T}{\partial \beta}=0,
$$

then we will have an expression for the left-hand side of expression (8) along the coordinate $\beta$ :

$$
\frac{d}{d t}\left(\frac{\partial T}{\partial \dot{\beta}}\right)-\frac{\partial T}{\partial \beta}=I_{B} \ddot{\beta} .
$$

The potential energy $P$ of the system is determined by the following expression:

$$
P=c\left(l_{1} \beta-y\right)^{2},
$$

where $c$-coefficient of rigidity of the pneumatic tires of the tracer wheels, $\mathrm{N} \cdot \mathrm{m}^{-1}$;

$l_{1}-$ distance from the suspension axis (point $B$ ) of the cleaner to the axis of the tracer wheels, $\mathrm{m}$.

The dissipative function $R$ of the particular dynamical system has the form:

$$
R=\mu\left(l_{1} \dot{\beta}-\dot{y}\right)^{2},
$$

where $\mu$-damping coefficient of the pneumatic tracer wheels, $\mathrm{N} \cdot \mathrm{s} \cdot \mathrm{m}^{-1}$.

In a similar way, the partial derivatives for (20) and (21) are equal to:

$$
\begin{aligned}
& \frac{\partial P}{\partial \beta}=2 c l_{1}\left(l_{1} \beta-y\right) \\
& \frac{\partial R}{\partial \dot{\beta}}=2 \mu l_{1}\left(l_{1} \dot{\beta}-\dot{y}\right) .
\end{aligned}
$$

For the second generalised coordinate $y$, we find similar expressions:

$$
\begin{gathered}
\frac{\partial T}{\partial \dot{y}}=m \dot{y} . \\
\frac{d}{d t}\left(\frac{\partial T}{\partial \dot{y}}\right)=m \cdot \ddot{y}, \\
\frac{\partial T}{\partial y}=0, \\
\frac{\partial P}{\partial y}=-2 c\left(l_{1} \beta-y\right), \\
\frac{\partial R}{\partial \dot{y}}=-2 \mu\left(l_{1} \dot{\beta}-\dot{y}\right) .
\end{gathered}
$$

Let us find generalised forces $Q_{\beta}$ and $Q_{y}$ with respect to the corresponding generalised coordinates $\beta$ and $y$, which enter the right-hand side of the Lagrange equation of the 2nd kind (8). For this purpose, in Fig. 4 we will show a change in the position of the cleaner when the tracer wheel is moved from the point $K_{1}$ which is the origin of the coordinate system $K_{1} X Y$, to the point $K_{2}$. The generalised force $Q_{\beta}$ 
from the generalised angular coordinate $\beta$ will have the dimension of the moment and will be determined using the expression for the elementary work of the forces on a possible angular displacement $\delta \beta[12]$ :

$$
\delta W_{\beta}=\delta W\left(M_{\text {rot. }}-M_{\text {res.rot. }}\right),
$$

where $M_{\text {rot. }}-$ moment of forces tending to rotate the cleaner frame around the axis that passes through the point $B, \mathrm{~N} \cdot \mathrm{m}$;

$M_{\text {res.rot }}$ - moment of forces resisting to the rotation of the cleaner frame around the axis that passes through the point $B, \mathrm{~N} \cdot \mathrm{m}$.

As it is evident from the scheme in Fig. 4, the turning moment of the cleaner frame around the axis passing through the point $B$ will create reaction forces of the soil surface $\bar{N}$ and $f \bar{N}$, as well as a force in the upper linkage of the mounting $\overline{S_{T}}$ and a resistance force $\overline{F_{r e s}}$.

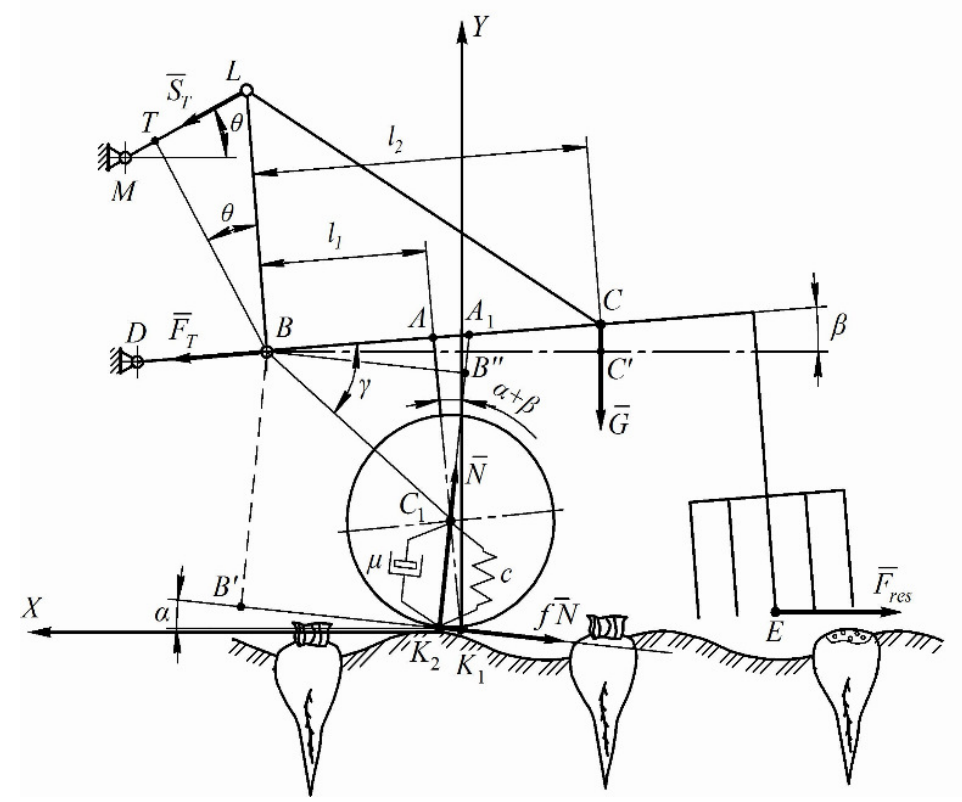

Fig. 4. Scheme for determining the moments of forces acting upon the cleaner

Then the total turning moment $M_{\text {rot. }}$ of the cleaner will be equal to:

$$
M_{\text {rot. }}=N \cdot B B^{\prime \prime}+f N \cdot B B^{\prime}+F_{\text {res }} \cdot H+S_{T} \cdot B T,
$$

where $B B^{\prime \prime}, B B^{\prime}, H$ and $B T-$ arms of the corresponding forces relative to the point $B$. Fig. 4.:

Let us define the indicated arms of the forces that enter into expression (30), using the scheme of

$$
B B^{\prime \prime}=l_{1} \cos (\alpha+\beta)+(H-r) \cdot \sin (\alpha+\beta),
$$

where $\alpha$-inclination angle of the tangent to the sinusoid (1).

As it is known, the tangent of the angle of inclination to the curve $y=f(x)$ at the particular point is equal to the derivative of the equation of this curve with respect to the variable $x$ at this point, that is: $\operatorname{tg} \alpha=y_{x}^{\prime}$.

Taking into account expression (1), we obtain:

$$
y_{x}^{\prime}=h k \cos \left(k x-\frac{\pi}{2}\right) .
$$

Therefore:

$$
\alpha=\operatorname{arctg}\left[h k \cos \left(k x-\frac{\pi}{2}\right)\right] .
$$


Further, from the scheme of Fig. 4 we will obtain:

$$
B B^{\prime}=r+\cos \left(90^{\circ}+\alpha+\beta-\gamma\right) \sqrt{(H-r)^{2}+l_{1}^{2}} .
$$

Besides:

$$
\begin{gathered}
\operatorname{tg} \gamma=\frac{A C_{1}}{A B}=\frac{H-r}{l_{1}}, \\
\gamma=\operatorname{arctg} \frac{H-r}{l_{1}} . \\
B T=B L \cdot \cos (\beta+\theta),
\end{gathered}
$$

where $B L$-constructive parameter, $\mathrm{m}$.

Substituting the obtained values of the arms of the forces (31), (35) and (38) into expression (30) and carrying out the corresponding transformations, we obtain:

$$
\begin{aligned}
& M_{\text {rot. }}=N\left[l_{1} \cos (\alpha+\beta)+(H-r) \sin (\alpha+\beta)\right]+f N[r+ \\
& \left.+\cos \left(90^{\circ}+\alpha+\beta-\gamma\right) \sqrt{(H-r)^{2}+l_{1}^{2}}\right]+F_{r e s} H+S_{T} B L \cos (\beta+\theta) .
\end{aligned}
$$

Let us determine the moment forces $M_{\text {res.rot }}$ resisting to the rotation of the cleaner frame around the axis passing through the point $B$ :

$$
M_{\text {res.rot. }}=G \cdot B C^{\prime},
$$

where $B C^{\prime}-\operatorname{arm}$ of forces $\bar{G}$ relative to the point $B$.

Using the scheme in Fig. 4 from the triangle $B C C^{\prime}$ we find the value of the arm $B C^{\prime}$ :

$$
B C^{\prime}=l_{2} \cos \beta .
$$

Consequently, the moment $M_{\text {res.rot }}$ of the resistance force $\bar{G}$ to the rotation of the cleaner frame relative to the axis passing through the point $B$ will finally be equal to:

$$
M_{\text {res.rot. }}=G \cdot l_{2} \cos \beta .
$$

Using the expressions (39) and (42) obtained and substituting their values into expression (29), we can finally determine $\delta W_{\beta}$ which will be equal to:

$$
\begin{aligned}
& \delta W_{\beta}=\left(M_{\text {rot. }}-M_{\text {res.rot. }}\right) \delta \beta=\left\{N\left[l_{1} \cos (\alpha+\beta)+(H-r) \sin (\alpha+\beta)\right]+\right. \\
& \left.+f N\left[r+\cos \left(90^{\circ}+\alpha+\beta-\gamma\right) \sqrt{(H-r)^{2}+l_{1}^{2}}\right]+F_{r e s} H-G l_{2} \cos \beta\right\} \delta \beta .
\end{aligned}
$$

From the expression (43) we find the generalised force $Q_{\beta}$ with respect to the angular coordinate $\beta$ :

$$
\begin{aligned}
& Q_{\beta}=\frac{\delta W_{\beta}}{\delta \beta}=N\left[l_{1} \cos (\alpha+\beta)+(H-r) \sin (\alpha+\beta)\right]+f N[r+ \\
& \left.+\cos \left(90^{\circ}+\alpha+\beta-\gamma\right) \sqrt{(H-r)^{2}+l_{1}^{2}}\right]+F_{r e s} H-G l_{2} \cos \beta .
\end{aligned}
$$

The generalised force $Q_{\beta}$ in this case is the algebraic sum of the moments of all active forces with respect to the point $B$. To determine it from the generalized coordinate $y$, let us use the expression for the elementary work of forces on displacement $\delta y$ : 


$$
\begin{aligned}
\delta W_{y}= & \sum_{i=1}^{n} Q_{y} \cdot \delta y=-G \cdot \delta y-f N \sin \alpha \cdot \delta y+F_{r e s} \sin \beta \cdot \delta y+ \\
& +N \cos \alpha \cdot \delta y-F_{T} \sin \beta \cdot \delta y-S_{T} \cdot \sin (\beta+\theta) \cdot \delta y .
\end{aligned}
$$

The normal $\bar{N}$ and tangential $f \bar{N}$ reactions of the soil on the tracer wheels perform the elementary work, respectively, on the deformation of the soil and overcoming friction, i.e. they are conditionally active forces. Hence, we have that the generalised force $Q_{y}$ will be equal to:

$$
\begin{aligned}
Q_{y}= & \frac{\delta W_{y}}{\delta y}=-G-f N \sin \alpha+F_{\text {res }} \sin \beta+ \\
& +N \cos \alpha-F_{T} \sin \beta-S_{T} \sin (\beta+\theta) .
\end{aligned}
$$

\section{Results and discussion}

Substituting the value of all the obtained components into the system (8), we obtain the following system of differential equations:

$$
\begin{aligned}
& I_{B} \ddot{\beta}=N\left[l_{1} \cos (\alpha+\beta)+(H-r) \cdot \sin (\alpha+\beta)\right]+f N[r+ \\
& \left.+\cos \left(90^{\circ}+\alpha+\beta-\gamma\right) \sqrt{(H-r)^{2}+l_{1}^{2}}\right]+F_{r e s} H-G l_{2} \cos \beta+ \\
& +S_{T} B L \cos (\beta+\theta)-2 c l_{1}\left(l_{1} \beta-y\right)-2 \mu l_{1}\left(l_{1} \dot{\beta}-\dot{y}\right), \\
& m \ddot{y}=-G-f N \sin \alpha+F_{r e s} \sin \beta+N \cos \alpha-F_{T} \sin \beta- \\
& -S_{T} \sin (\beta+\theta)+2 c\left(l_{1} \beta-y\right)+2 \mu\left(l_{1} \dot{\beta}-\dot{y}\right) .
\end{aligned}
$$

After transformations have been performed, we obtain a system of the two equations, which is a mathematical model of the movement of the sugar beet root cleaner hanged from behind to the wheeled integral row-crop tractor:

$$
\left.\begin{array}{l}
\ddot{\beta}=\frac{N\left[l_{1} \cos (\alpha+\beta)+(H-r) \sin (\alpha+\beta)\right]}{I_{B}}+ \\
+\frac{f N\left[r+\cos \left(90^{\circ}+\alpha+\beta-\gamma\right) \sqrt{(H-r)^{2}+l_{1}^{2}}\right]}{I_{B}}+ \\
+\frac{F_{r e s} H-G l_{2} \cos \beta+S_{T} B L \cos (\beta+\theta)-2 c l_{1}\left(l_{1} \beta-y\right)-2 \mu l_{1}\left(l_{1} \dot{\beta}-\dot{y}\right)}{I_{B}}, \\
\ddot{y}=\frac{-G-f N \sin \alpha+F_{r e s} \sin \beta+N \cos \alpha-F_{T} \sin \beta-S_{T} \sin (\beta+\theta)}{m}+ \\
+\frac{2 c\left(l_{1} \beta-y\right)+2 \mu\left(l_{1} \dot{\beta}-\dot{y}\right)}{m} .
\end{array}\right\}
$$

The obtained system of equations (48) includes an unknown normal reaction $\bar{N}$, as well as the resistance force $\overline{F_{\text {res }}}$ to the movement of the cleaning tool along the head of the beet root. A normal reaction $\bar{N}$ can be determined from the equilibrium condition of the system at a fixed time (the equation of statics). It can be obtained from equation (44), provided that $Q_{\beta}=0$ : 


$$
\begin{aligned}
& N\left[l_{1} \cos (\alpha+\beta)+(H-r) \sin (\alpha+\beta)\right]+ \\
& +f N\left[r+\cos \left(90^{\circ}+\alpha+\beta-\gamma\right) \sqrt{(H-r)^{2}+l_{1}^{2}}\right]+ \\
& +F_{r e s} H-G l_{2} \cos \beta+S_{T} B L \cos (\beta+\theta)=0 .
\end{aligned}
$$

When the reaction $\bar{N}$ is determined, the unknown angle $\beta$ can be assumed sufficiently small in the first approximation and considered to be equal to zero. Then the expression (49) obtains an appearance:

$$
\begin{aligned}
& N\left[l_{1} \cos \alpha+(H-r) \sin \alpha\right]+ \\
& +f N\left[r+\cos \left(90^{\circ}+\alpha-\gamma\right) \sqrt{(H-r)^{2}+l_{1}^{2}}\right]+ \\
& +F_{\text {res }} H-G l_{2}+S_{T} B L \cos \theta=0 .
\end{aligned}
$$

From the expression (50) we will define the initial reaction $N$, which will be:

$$
N=\frac{G l_{2}-F_{r e s} H-S_{T} B L \cos \theta}{l_{1} \cos \alpha+(H-r) \sin \alpha+f\left[r+\cos \left(90^{\circ}+\alpha-\gamma\right) \sqrt{(H-r)^{2}+l_{1}^{2}}\right]} .
$$

Expression (51) is obtained for the case when the tracer wheel is at an arbitrary point of the sinusoidal profile of the soil surface irregularity (1). If the tracer wheel is on a horizontal flat surface (axis $\left.K_{1} X\right)$, the expression (51) will become even simpler $(\alpha=0)$, and the normal reaction $N$ will, in this case, be equal to:

$$
N=\frac{G l_{2}-F_{r e s} \cdot H-S_{T} B L \cos \theta}{l_{1}+f\left[r+\sin \gamma \sqrt{(H-r)^{2}+l_{1}^{2}}\right]} .
$$

The value of the normal reaction $N$ determined from the formula (52) with sufficient accuracy for practice can be used to solve the system of equations (48).

The force of resistance $F_{\text {res }}$ to the movement of the cleaning tool along the head of the sugar beet root is determined from the condition of its equality in terms of the stripping strength $\overline{Q_{b r u}}$ of the remnants of the foliage. The process of stripping the remnants of the foliage from the beet root head is possible under the following condition:

$$
\frac{\bar{Q}_{b r u}}{n F} \geq[\tau],
$$

where $\overline{Q_{b r u}}$ - stripping force;

$[\tau]$ - allowed tangential shear stresses for the stalk of the foliage;

$F$ - cross-sectional area of one stalk of the foliage;

$n$ - number of stalks, which are simultaneously stripped.

Now we will compute the stripping force $\overline{Q_{b r u}}$, necessary for stripping the leaves of the tops from the beet root head. From condition (53) we obtain:

$$
\bar{Q}_{b r u} \geq n F[\tau] .
$$

The cross-sectional area of the stalk of the beet top will be [7]:

$$
F=a h-a_{o} h_{o},
$$

where $h$-height of the root crop;

$a$ - radius in the root crop in its upper part;

$a_{o}$ - radius of the stalk in its upper part;

$h_{o}-$ height of the stalk.

Taking into account expressions (54) and (55), we find the stripping force $\overline{Q_{b r u}}$ : 


$$
\bar{Q}_{b r u} \geq\left(a h-a_{o} h_{o}\right) n[\tau],
$$

Let us determine the force $\overline{Q_{b r u}}$, necessary for stripping one stalk of the foliage, which, according to [10], has such linear dimensions: $a=5 \mathrm{~mm} ; a_{o}=2 \mathrm{~mm} ; h=5 \mathrm{~mm} ; h_{o}=2 \mathrm{~mm}$.

The average value of the allowable tangential stress $[\tau]$ for the stalk material, also according to [10], will be equal to: $[\tau]=1.14 \cdot 10^{6} \mathrm{~Pa}$.

Substituting these values into expression (56) at $n=1$, we obtain:

$$
\bar{Q}_{b r u} \geq(5 \cdot 5-2 \cdot 2) \cdot 10^{-6} \cdot 1.14 \cdot 10^{-6}=23.9 \mathrm{~N}
$$

Considering that the stems of the tops may have dimensions in the cross section that are somewhat larger than the average, we take $\overline{Q_{b r u}}=25 \mathrm{~N}$. According to the experimental data [4], in order to satisfactorily carry out the stripping process of the remnants of the tops from the surface of the root crop head without its extraction from the soil, tangential forces within a range from $70-120 \mathrm{~N} \cdot \mathrm{cm}^{-2}$ are required. Since the cross-sectional area of one stalk is, on the average, $F=0.25 \mathrm{~cm}^{2}$, we find that for stripping one stem of the foliage, the necessary tangential force is within the range $17.5-30 \mathrm{~N}$. Thus the value of the stripping force $\overline{Q_{b r u}}$, obtained theoretically for one stalk, falls precisely into this interval. We will assume that in one pass the cleaning blade strips (combs) a number of the remnants of the foliage, consisting of four upstanding stalks (i.e. $n=4$ ), from the expression (57) we finally obtain:

$$
\bar{Q}_{b r u} \geq 95.6 \mathrm{~N} .
$$

For practical calculations in the system of equations (48) we can assume $\overline{Q_{b r u}}=100 \mathrm{~N}$.

In order to solve the system of equations (48), it is necessary to specify the constructive, kinematic and force parameters included in this system. First of all, we define the initial conditions that the initial variables satisfy:

$$
t=0: y=0, \dot{y}=0, \beta=0 \text { and } \dot{\beta}=0 .
$$

Further, we present the necessary data for numerical calculations:

- design parameters of the machine: $l_{1}=0.6 \mathrm{~m} ; l_{2}=0.75 \mathrm{~m} ; r=0.20 \mathrm{~m} ; H=0.43 \mathrm{~m}$.

- kinematic parameters: $V_{M}=2.0 \mathrm{~m} \cdot \mathrm{s}^{-1}$.

- inertial characteristics: $m=11 \mathrm{~kg} ; I_{B}=25 \mathrm{~kg} \cdot \mathrm{m}^{2}$.

- characteristics of the elastic properties of the tires: $C=2000-2500 \mathrm{~N} \cdot \mathrm{m}^{-1} ; \mu=73-85 \mathrm{~N} \cdot \mathrm{s} \cdot \mathrm{m}^{-1}$.

- power characteristics: $G=700 \mathrm{~N} ; P=1000 \mathrm{~N} ; N=651.6 \mathrm{~N}$ (formula (52)); $f=0.2$.

- characteristics of the irregularities of the soil surface: $h=0.08 \mathrm{~m} ; k=1.75 \mathrm{~m}^{-1}$.

- angular parameters: $\gamma=0.437 \mathrm{rad}$ (formula (37)).

- $\alpha=\operatorname{arctg}\left[h k \cos \left(k V_{M} t-\frac{\pi}{2}\right)\right]-$ a variable angle (it is substituted into the system of equations (48) in the form of the indicated formula).

After compiling a program on the PC (in Mathcad), numerical calculations of the values of the angle $\beta$ and the coordinate $y$ were performed depending on the design parameters of the root head cleaner itself from the remnants of the tops without extracting the heads from the soil (Figs 6-8).

The graph (Fig. 6) shows the dependence of vertical oscillations $y$ of the tracer wheels of the considered cleaner as a function of time $t$ when it moves along the irregularities of the soil surface. The thin line indicates the path (trajectory) of the soil surface irregularity itself. It follows from the graph that, in order to ensure minimum vertical oscillations, the tracer wheels of the root head cleaner $y$ must have a radius $r$ that is not more than $0.20 \mathrm{~m}$ (further reduction of the radius does not lead to a significant change in the magnitude of the oscillations).

The dependence of the vertical oscillations $y$ on time $t$ at different values of the stiffness coefficient $C$ of pneumatic tires of the wheels (Fig. 7) shows that its reduction within the allowed limits will also ensure minimum values of the vertical oscillations yin the case of using the previously defined radius $r$ of the tracer wheels. 


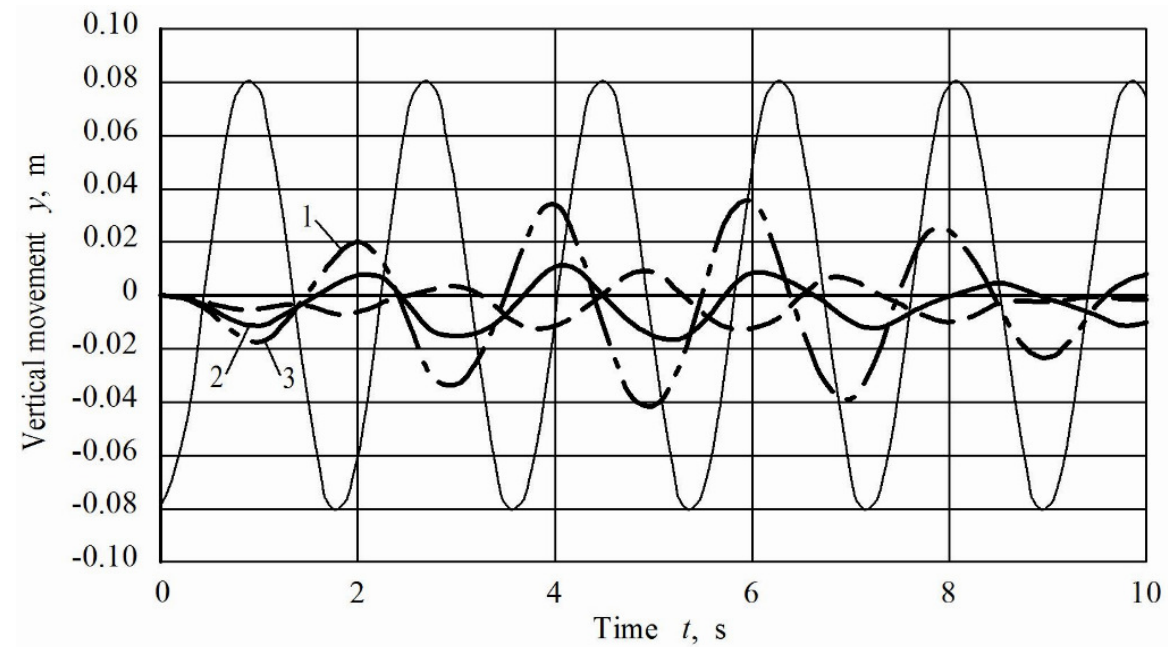

Fig. 6. Dependence of vertical oscillations on time $t$ at different values of the radius $r$ of the tracer wheels: $1-r=0.25 \mathrm{~m} ; 2-r=0.20 \mathrm{~m} ; 3-r=0.15 \mathrm{~m}$

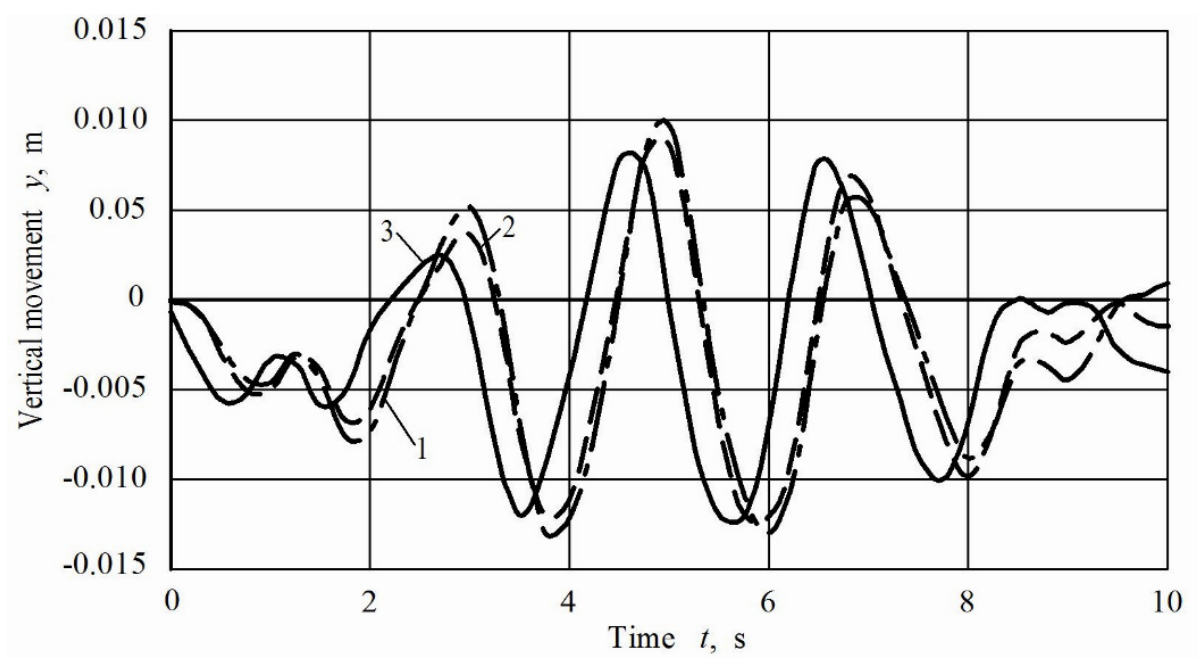

Fig. 7. Dependence of vertical oscillations $y$ on time $t$ at different values of the stiffness coefficient $C$ of pneumatic tires of the wheels of the copying system: $1-C=2500 \mathrm{~N} \cdot \mathrm{m}^{-1}$;

$$
2-\mathrm{C}=2200 \mathrm{~N} \cdot \mathrm{m}^{-1} ; 3-C=2000 \mathrm{~N} \cdot \mathrm{m}^{-1}
$$

Fig. 8 shows the dependence of vertical oscillations $y$ on time $t$ at different values of distance $l_{2}$ from the suspension point of the cleaner to the centre of its masses (point $C$ ).

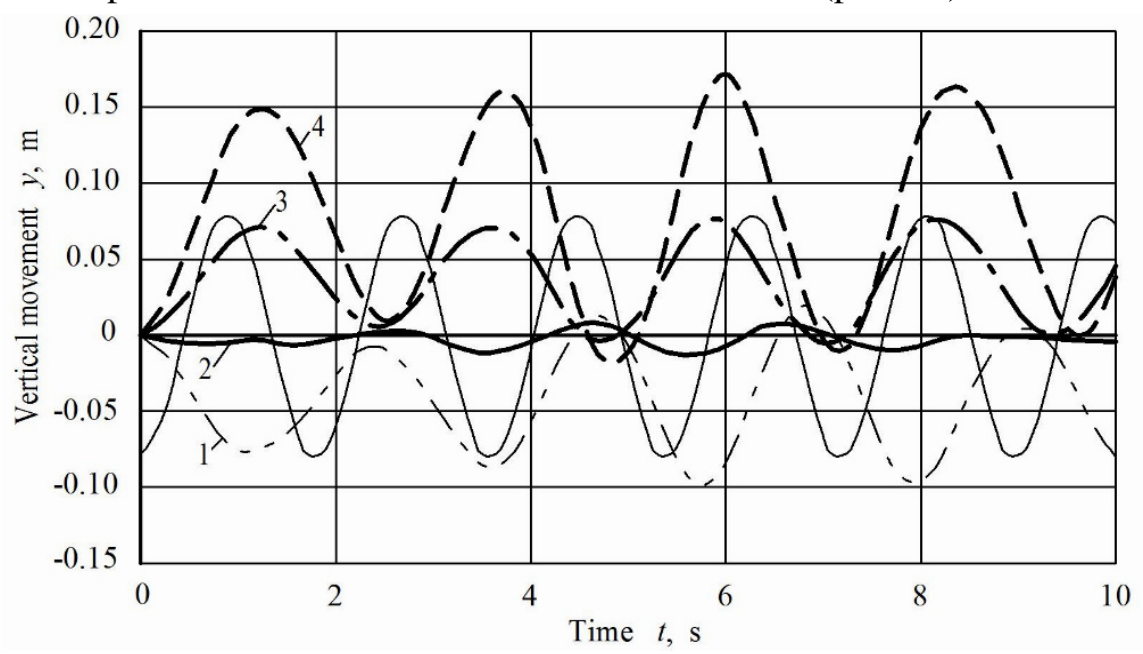

Fig. 8. Dependence of the magnitude of vertical oscillations $y$ on time $\boldsymbol{t}$ at different values of the distance $l_{2}: 1-l_{2}=0.70 \mathrm{~m} ; 2-l_{2}=0.75 \mathrm{~m} ; 3-l_{2}=0.80 \mathrm{~m} ; 4-l_{2}=0.85 \mathrm{~m}$ 
The most significant factor that affects the magnitude of the vertical oscillations $y$ of the tracer wheels is the distance $l_{2}$ from the suspension point of the cleaner to the centre of its masses (point $C$ ). In the case when it is equal to $0.75 \mathrm{~m}$, with other design and power parameters of the cleaner, which are also accepted as rational, the oscillations of the tracer wheels are insignificant and tend to zero. Increasing the distance $l_{2}$ the oscillations increase sharply, which adversely affects the quality of the technological process. If it turns out that the distance $l_{2}$ is less than the optimal $0.75 \mathrm{~m}$, then the displacement of the oscillations towards the negative values is observed, which leads to additional pressing of the copying wheels to the surface of the agrophone and can be used to reduce the oscillations of the cleaner.

On the basis of numerical simulation on the PC, it has been established that the most rational kinematic and design parameters of the sugar root beet head cleaner without extracting the heads from the soil, which ensure high quality indicators of cleaning the heads from the tops and with minimum oscillations of the operating tool in the longitudinally vertical plane, are: the radius $r$ of the tracer wheels is not more than $0.2 \mathrm{~m}$, the stiffness coefficient $c$ of the pneumatic tires of the wheels of the copying system is not more than $2000 \mathrm{~N} \cdot \mathrm{m}^{-1}$, and the distances $l_{2}$ from the suspension point of the cleaner to the centre of its masses $-0.75 \mathrm{~m}$.

\section{Conclusions}

1. A design of a sugar beet head cleaner from the remains of the tops without extracting the roots from the soil has been developed, which has a vertical drive shaft with cleaning tools mounted on its end face, and pneumatic tracerwheels. A system of two nonlinear differential equations has been built for this structure that describe the oscillations of the root crop head cleaner in the longitudinal-vertical plane when its pneumatic tracer wheels are moving along the irregularities of the soil surface.

2. It has been established that the most significant factor affecting the magnitude of vertical oscillations of the tracer wheels in a longitudinal-vertical plane (for the given design parameters) is the distance from the suspension point of the cleaner on the aggregating tractor to the centre of its masses.

3. As a result of numerical simulation, some rational design parameters of the root head cleaner have been determined: the radius $r$ of the tracer wheels is not more than $0.2 \mathrm{~m}$, the stiffness coefficient $c$ of the pneumatic tires of the wheels of the copying system is not more than $2000 \mathrm{~N} \cdot \mathrm{m}^{-1}$, and the distance $l_{2}$ from the point of the cleaner suspension to the centre of its masses is $0.75 \mathrm{~m}$.

\section{References}

1. Ivančan S., Sito S., Fabijanić G. Factors of the quality of performance of sugar beet combine harvesters. Bodenkultur, No 53(3), 2002. pp. 161-166.

2. Bulgakov V., IvanovsS., Adamchuk V., Boris A. Mathematical model for determination of losses of sugar bearing-mass when sugar beet tops are removed. Engineering for Rural Development, Proceedings, Vol. 14, 2015. pp. 41-45

3. Василенко П. Введение в земледельческую механику (Introduction to agricultural mechanics.). Kyiv, 1996. 252 p. (In Ukrainian).

4. Chochola J. New trial beet harvesting machine. Listy Cukovarnicke a Reparske. Vol.128 (5-6), 2012, pp.174-178.

5. Lammers $P$. Harvest and loading machines for sugar beet. International sugar journal.

6. Vol. 113 (1348), 2011, pp. 253-256.

7. Markus D., Braunhuber R., Gescheder R. Effect of heave agricultural machines for sugar beet harvesting. American Society of Agricultural and Biological Engineers Annul International Meeting ASABE 2008. Vol.10, 2008, pp. 6143-6151.

8. Bulgakov V., Ivanovs S., Ruzhylo Z., Golovach I. Theoretical investigations in cleaning sugar beet heads from remnants of leaves by cleaning blade. Engineering for Rural Development, Proceedings, 2016, Vol. 15, pp. 1090-1097.

9. Гевко Р. и др. Направления совершенствования свеклоуборочных машин (Directions for the improvent of beet harvesters). Луцк, 1999, 170 p.(In Ukrainian). 
10. Хелемендик Н.M. Повышение механико-технологической эффективности трудоемких процессов в свекловодстве (Increasing the Mechanical and Technological Efficiency of the Labour Consuming Processes in Beat Growing). Ternopol, 1996. 48 p. (In Ukrainian).

11. Bulgakov V., Adamchuk V., Arak M., Nadykto V., Kyurchev V., Olt J. Theory of vertical oscillations and dynamic stability of combined tractor-implement unit. Agronomy Research. 2016, vol.14. No 3. pp. 689-710.

12. Колычев Е., Перельцвайг И. О выборе расчетного случая воздействия при исследовании плавности хода тракторов и сельхозмашин (On the choice of a calculated of impact in the study of the smooth running of tractors and agricultural machines), Тракторы и сельхозмашины. 1976, No 3. pp. 9-11. (In Russian).

13. Dreizler R.M., Ludde C.S. Theoretical Mechanics. Springer, 2010, 402 p. 